\title{
Processamento auditivo em gagos: análise do desempenho das orelhas direita e esquerda
}

\section{Auditory processing in stutterers: performance of right and left ears}

\author{
Adriana Neves de Andrade ${ }^{1}$, Daniela Gil ${ }^{2}$, Ana Maria Schiefer ${ }^{3}$, Liliane Desgualdo Pereira ${ }^{4}$
}

\begin{abstract}
RESUMO
Objetivo: Comparar a diferença entre as orelhas nos testes comportamentais do processamento auditivo e os resultados de sujeitos com diferentes graus de gravidade de gagueira em cada teste do processamento auditivo. Métodos: Cinqüenta e seis indivíduos, com idades entre quatro e 34 anos, foram encaminhados pelo Ambulatório de Avaliação Fonoaudiológica da UNIFESP para avaliação comportamental do processamento auditivo. Todos os pacientes foram submetidos à avaliação de audição, fala e linguagem. A disfluência foi classificada segundo o protocolo de Riley (1994), o qual prevê os seguintes graus de gravidade da gagueira: muito leve, leve, moderado, severo e muito severo. Os testes para avaliação do processamento auditivo foram selecionados e analisados de acordo com a idade do paciente e a proposta de Pereira \& Schochat (1997). Resultados: Observamos prevalência da gagueira de grau leve nas faixas etárias de quatro a sete anos e de 12 a 34 anos de idade, e de grau moderado nos indivíduos de oito a 11 anos de idade. Dos 56 indivíduos avaliados $92,85 \%$ apresentaram alteração do processamento auditivo. Houve diferença estatisticamente significante entre as orelhas direita e esquerda na etapa de atenção direcionada do teste dicótico não verbal, em todas as faixas etárias estudadas. Não foram encontradas diferenças significativas entre os graus de gravidade da gagueira em nenhum dos testes de processamento auditivo. Conclusões: A orelha direita apresentou melhor desempenho do que a esquerda nos diferentes testes comportamentais. O grau de gravidade da gagueira não interferiu no resultado de cada teste.
\end{abstract}

DESCRITORES: Gagueira; Transtornos da percepção auditiva; Audição; Transtornos da fala

\section{INTRODUÇÃO}

A fluência verbal pode ser definida como a fala de fluxo contínuo e suave decorrente de uma integração harmônica entre os processamentos neurais envolvidos na linguagem e no ato motor ${ }^{(1-2)}$.

Para que a fala seja fluente os sistemas simbólicos e de sinais, devem estar equilibrados temporalmente antes que a mensagem gerada chegue ao córtex motor ${ }^{(3)}$. Se estes sistemas desequilibram-se o fluxo da fala é temporariamente rompido,

Este trabalho foi realizado no Ambulatório de Avaliação do Processamento Auditivo da Disciplina dos Distúrbios da Audição do Departamento de Fonoaudiologia da Universidade Federal de São Paulo - UNIFESP - São Paulo (SP), Brasil.

(1) Pós-graduanda em Distúrbios da Comunicação Humana - Campo Fonoaudiológico da Universidade Federal de São Paulo - UNIFESP - São Paulo (SP), Brasil.

(2) Doutora, Professora Adjunto do Departamento de Fonoaudiologia da Universidade Federal de São Paulo - UNIFESP - São Paulo (SP), Brasil.

(3) Doutora; Professora Adjunto do Departamento de Fonoaudiologia da Universidade Federal de São Paulo - UNIFESP - São Paulo (SP), Brasil.

(4) Doutora, Professora Adjunto do Departamento de Fonoaudiologia da Universidade Federal de São Paulo - UNIFESP - São Paulo (SP), Brasil.

Endereço para correspondência: Adriana Neves de Andrade. R. São Francisco, 498, Santo Antônio, São Caetano do Sul - SP, CEP 09530-050. E-mail: adriandrad34@yahoo.com.br

Recebido em: 21/6/2007; Aceito em: 16/12/2007 gerando as disfluências ${ }^{(4)}$.

Assim, a disfluência é um distúrbio que ocorre no momento da produção da fala, na qual o falante se depara com um impedimento que o impossibilita, momentaneamente, de produzir a palavra que deseja falar ${ }^{(5)}$.

Existe uma redução na habilidade de produção dos padrões de percepção auditiva em indivíduos gagos, quando comparados aos não gagos ${ }^{(6)}$. A imprecisão temporal na percepção de fala pode levar a momentos de disfluência e a diminuição das habilidades de processamento pode estar relacionada à incapacidade de manutenção da fala fluente ${ }^{(7-8)}$.

Denominamos processamento auditivo (PA) o conjunto de habilidades específicas das quais o indivíduo depende para interpretar o que ouve ${ }^{(9)}$. Quando o indivíduo perde parcialmente ou totalmente a função da análise das imagens auditivas, estamos diante de um distúrbio do processamento auditivo.

O PA pode ser um dos fatores que contribuem para a produção do discurso disfluente no nível da produção da sílaba. A relação entre a fluência verbal e a alteração do processamento auditivo não é um fenômeno relacionado somente à gagueira ou não gagueira, mas também um fenômeno normal de fluência e não fluência ${ }^{(10)}$.

Existe a hipótese de que os indivíduos com gagueira possuem alteração na dominância cerebral da função da linguagem. Investigações realizadas sobre o desempenho das 
orelhas direita e esquerda em testes comportamentais do PA em indivíduos gagos e não gagos, não revelaram diferenças entre os grupos estudados e nem entre os graus de gravidade da gagueira e alterações do PA, apesar do desempenho dos indivíduos gagos ter sido discretamente pior em comparação com os não gagos. Desta forma, não foi possível concluir sobre diferenças entre a dominância cerebral hemisférica para a fala, habilidades receptivas e a gagueira ${ }^{(11)}$.

Tendo em vista a correlação entre o processamento auditivo e a gagueira, este estudo tem por objetivos:

Comparar a diferença entre as orelhas direita e esquerda nos testes comportamentais do processamento auditivo em indivíduos gagos.

Comparar os resultados de cada teste do processamento auditivo com o grau de gravidade da gagueira.

\section{MÉTODOS}

Este trabalho foi analisado e aprovado pelo comitê de ética em pesquisa da Universidade Federal de São Paulo sob o n ${ }^{\circ}$ 0745/05. Realizou-se um estudo retrospectivo por meio do levantamento de prontuários de 56 indivíduos com alteração da fluência da fala, avaliados durante o período de 1999 a 2005, no ambulatório de Avaliação e Diagnóstico Fonoaudiológico da Disciplina dos Distúrbios da Comunicação Humana do Departamento de Fonoaudiologia da Universidade Federal de São Paulo e encaminhados para a avaliação comportamental do Processamento Auditivo na mesma instituição.

Nesta pesquisa, todos os indivíduos foram submetidos à avaliação fonoaudiológica específica de gagueira, avaliação audiológica básica e comportamental do processamento auditivo. Analisamos os dados dos protocolos considerando às variáveis: faixa etária, tipo e grau de disfluência da fala.

Da avaliação fonoaudiológica, utilizamos, para este estudo, apenas a classificação do grau de gravidade da gagueira, que foi realizado segundo o Stuttering Severity Instrument for Children and Adults $-S S I-3^{(12)}$. Este instrumento classifica a gagueira em: grau muito leve, leve, moderado, severo e muito severo; considerando a freqüência e duração das rupturas da fala, bem como a presença de concomitantes físicos associados a essas rupturas, por meio da análise da fala espontânea e leitura (apenas para leitores). Neste procedimento, foi adotado como critério para gagueira a presença de, no mínimo, $3 \%$ de disfluências atípicas. Foram consideradas disfluências atípicas as seguintes manifestações: repetição de sílabas, repetição de sons, prolongamento, bloqueio, repetição de palavra (três ou mais).

O grau de gravidade da gagueira segundo a faixa etária está ilustrado na Figura 1.

Optamos por não separar os gêneros para estudar a avaliação comportamental do processamento auditivo, pois segundo outros autores esta variável não traz consequiências relevantes na avaliação do distúrbio do processamento auditivo ${ }^{(13-14)}$.

Em relação à idade, a amostra foi constituída por indivíduos de quatro a 34 anos de idade, com idade média de 12,35 anos. A população foi distribuída em três grupos etários: Grupo I - quatro a sete anos de idade (N=16), Grupo II - oito a 11 anos de idade $(\mathrm{N}=16)$ e Grupo III - maior que 12 anos de idade $(\mathrm{N}=24)$.

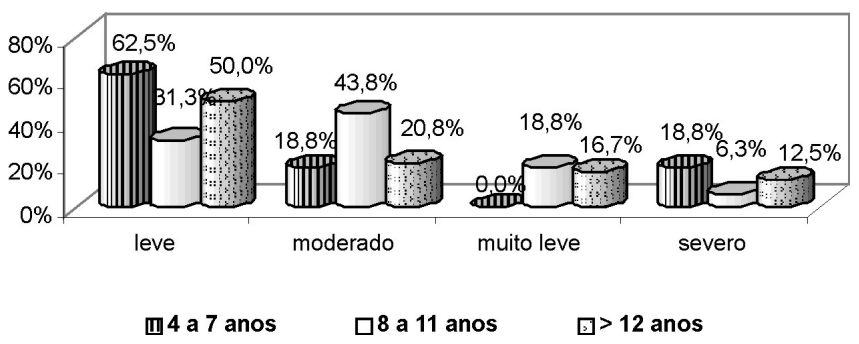

Figura 1. Distribuição do grau de gravidade da gagueira por faixa etária

A população foi separada em três faixas etárias, uma vez que a idade e o efeito da neuromaturação da via auditiva podem interferir com os resultados da avaliação comportamental do processamento auditivo ${ }^{(15)}$, sendo necessário selecionar e analisar os testes de acordo com a idade do paciente ${ }^{(16)} \mathrm{e}$ utilizar padrões de normalidade para cada faixa etária.

Antes da avaliação comportamental do PA foi realizada a avaliação audiológica básica, constituída por: anamnese, meatoscopia, audiometria tonal liminar, logoaudiometria, timpanometria e pesquisa dos reflexos acústicos contralaterais.

Todos os indivíduos avaliados apresentaram boa capacidade de detecção de tons puros e transmissão sonora adequada pelo sistema tímpano-ossicular, traduzidos por limiares auditivos inferiores a $25 \mathrm{~dB}$ NA entre 250 e $8000 \mathrm{~Hz}$ e curvas timpanométricas do tipo A e reflexos acústicos modo contralateral presentes em níveis adequados de intensidade.

Os testes comportamentais especiais para a avaliação do PA foram realizados em cabina acústica, utilizando-se estímulos não verbais e verbais gravados em compact disc, apresentados por meio de fones auriculares (TDH-39) acoplados a um audiômetro de dois canais (Interacoustics AC33), calibrados de acordo com a norma ISO 8253-1 (1989).

Os testes realizados bem como as habilidades auditivas envolvidas e os padrões de normalidade por faixa etária estão apresentados no Quadro 1.

Os dados referentes aos testes da avaliação comportamental do processamento auditivo e grau de gravidade da gagueira foram registrados e analisados estatisticamente.

Para a análise estatística dos resultados foram aplicados os seguintes testes: Teste de Wilcoxon, Teste de Kruskal-Wallis e Teste de Igualdade de duas Proporções.

Para complementar a análise descritiva, fizemos uso também da técnica de intervalo de confiança, tanto para média quanto proporção.

Definimos para este trabalho um nível de significância de $0,10(10 \%)$, o qual é indicado para pequenas amostragens. Todos os intervalos de confiança obtidos ao longo do trabalho foram construídos com $95 \%$ de confiança estatística. Os resultados com significância estatística serão destacados com o símbolo asterisco (*) e os que tendem a ser significativos serão destacados com o símbolo sustenido (\#).

\section{RESULTADOS}

Para facilitar a leitura separamos os resultados em partes: comparação do número de respostas corretas das orelhas 
Quadro 1. Descrição da tarefa auditiva, habilidade auditiva e valores de normalidade de cada teste utilizado na avaliação comportamental do processamento auditivo

\begin{tabular}{|c|c|c|c|c|}
\hline Teste & Idade & Tarefa auditiva & Habilidade auditiva & Valores de normalidade ( $\%$ e/ou ${ }^{\circ}$ de acertos) \\
\hline TFRB & 7 anos & Monótica & Fechamento & $>=70 \%$ acertos e IPRF - TFRB $<20 \%$ \\
\hline $\mathrm{PSI}-40$ & 3 anos & Dicótica & Figura-fundo & $>=90 \%$ acertos \\
\hline PSI - 10 & 3 anos & Monótica & Figura-fundo & $>=70 \%$ acertos \\
\hline \multirow[t]{3}{*}{ PSI-PAL } & 4 anos & Monótica & Fechamento & $>=90 \%$ acertos \\
\hline & & & & Classificação de grau para menores de 7 anos: \\
\hline & & & & Leve: 80 a $89 \%$ Moderado: 60 a $79 \%$ Severo: $<60 \%$ \\
\hline SSI -40 & 8 anos & Dicótica & Figura-fundo & $>=90 \%$ acertos \\
\hline SSI - 10 & 8 anos & Monótica & Figura-fundo & $>=70 \%$ acertos \\
\hline TPF & 11 anos & Monótica & Aspectos temporais & $>12$ anos: $>=76 \%$ acertos \\
\hline TPD & 11 anos & Monótica & Aspectos temporais & $>12$ anos: $>=83 \%$ acertos \\
\hline TDNV & 5 anos & Dicótica & Figura-fundo & $\begin{array}{l}\text { Atenção livre } \mathrm{OD}=\mathrm{OE}=12 \text { (variação } 10-14 \text { e ter } 1 \text { erro) } \\
\text { Escuta direcionada ( } 7 \text { anos): >= } 22 \text { acertos } \\
\text { Escuta direcionada ( } 8 \text { anos): >= } 23 \text { acertos }\end{array}$ \\
\hline TDCV & 8 anos & Dicótica & Figura-fundo & $\begin{array}{l}\text { Atenção livre: > }=19 \text { acertos (OD > OE) } \\
\begin{aligned} 10 \text { a } 12 \text { anos - } & \text { EDD: } 1 \text { acerto a mais que na OD na atenção livre } \\
& \text { EDE: } 2 \text { acertos a mais que na OE na atenção livre } \\
>12 \text { anos - } & \text { EDD: } 2 \text { acertos a mais que na OD na atenção livre } \\
& \text { EDE: } 4 \text { acertos a mais que na OE na atenção livre }\end{aligned}\end{array}$ \\
\hline TDD & 5 anos & Dicótica & Figura-fundo & $\begin{array}{l}5 \text { a } 6 \text { anos: Atenção livre: OD >= } 81 \% \text { e OE >= } 74 \% \\
7 \text { a } 8 \text { anos: Atenção livre: OD >= } 85 \% \text { e OE >= } 82 \% \\
\text { > } 9 \text { anos: Atenção livre: OD = OE >= 95\% }\end{array}$ \\
\hline SSW & 6 anos & Dicótica & $\begin{array}{l}\text { Figura-fundo } \\
\text { Ordenação temporal }\end{array}$ & $\begin{array}{l}6 \text { anos: Direita Competitiva >= } 70 \% \text { Esquerda Competitiva }>=55 \% \\
7 \text { anos: Direita Competitiva >= } 75 \% \text { Esquerda Competitiva }>=65 \% \\
8 \text { anos: Direita Competitiva >= } 80 \% \text { Esquerda Competitiva >= } 75 \% \\
\text { >= } 9 \text { anos: Direita Competitiva >= 90\% Esquerda Competitiva >= } 90 \\
\text { Classificação quanto ao grau } \\
\text { Normal: }>90 \% \text { Leve: } 80-89 \% \text { Moderado: } 60-79 \% \text { Severo: } 0-59 \%\end{array}$ \\
\hline
\end{tabular}

Legenda: TRFB - Teste de Fala com Ruído Branco; IPRF - Índice Percentual de Reconhecimento de Fala com gravação; PSI-40 - Teste de Identificação de Sentenças Pediátricas (mensagem competitiva contralateral-relação fala/ruído -40dB); PSI-10 - Teste de Identificação de Sentenças Pediátricas (mensagem competitiva ipsilateral -relação fala/ruído -10dB); PSI-PAL - Teste de Identificação de Palavras com Ruído Branco; SSI-40 - Teste de Identificação de Sentenças Sintéticas (mensagem competitiva contralateral-relação fala/ruído -40dB); SSI-10 - Teste de Identificação de Sentenças Sintéticas (mensagem competitiva ipsilateral -relação fala/ruído -10dB); TPF - Teste de Reconhecimento de Padrão de Freqüência; TPD - Teste de Reconhecimento de Padrão de Duração; TDNV - Teste Dicótico Não Verbal; TDCV - Teste Dicótico Consoante Vogal; EDD - Escuta Direcionada à Direita; OD - Orelha Direita; EDE - Escuta Direcionada à Esquerda; OE - Orelha Esquerda; TDD - Teste Dicótico de Dígitos; SSW - Teste Dicótico de Dissílabos Alternados

direita e esquerda para cada teste do processamento auditivo realizado, segundo a variável faixa etária e comparação do número de respostas corretas nos testes do processamento auditivo nas diferentes faixas etárias, segundo a variável grau de gravidade da gagueira.

A avaliação do processamento auditivo apresentou-se alterada em 52 indivíduos $(92,85 \%)$. Os indivíduos que apresentaram adequação nos testes do processamento auditivo estavam distribuídos nas faixas etárias de quatro a sete anos (um indivíduo) e > 12 anos (três indivíduos).

Parte 1. Comparação do número de respostas corretas das orelhas direita e esquerda para cada teste do processamento auditivo, segundo a variável faixa etária

Nessa parte comparamos os acertos da orelha direita versus esquerda para os testes especiais para avaliar o PA que possuem amostragem significativa, em cada uma das faixas etárias (Tabelas 1, 2 e 3). Utilizamos nestas comparações o teste estatístico de Wilcoxon, pois temos dados pareados, ou seja, o mesmo indivíduo é pesquisa e controle dele mesmo.

Parte 2: Comparação do número de respostas corretas nos testes do processamento auditivo nas diferentes faixas etárias, segundo a variável grau de gravidade da gagueira

Iniciamos esta parte comparando entre as faixas etárias a proporção de cada um dos graus de gravidade da gagueira. Para tanto, utilizamos o teste de Igualdade de Duas Proporções, o qual é indicado para comparar a proporção de respostas de duas determinadas variáveis e/ou significância estatística entre seus níveis (Quadro 2).

Em seguida, comparamos os resultados dos testes do processamento auditivo entre os diferentes graus de gravidade da gagueira, considerando os resultados de ambas as orelhas (Tabelas 4, 5 e 6). Para tal, utilizamos o teste de Kruskal-Wallis, pois temos dados independentes com mais de dois níveis de 
Tabela 1. Medidas descritivas das respostas corretas das orelhas direita e esquerda para os testes de avaliação comportamental do processamento auditivo na faixa etária de quatro a sete anos

\begin{tabular}{|c|c|c|c|c|c|c|c|c|c|c|}
\hline 4 a 7 anos & & Média & Mediana & $\begin{array}{l}\text { Desvio } \\
\text { Padrão }\end{array}$ & Mínimo & Máximo & Tamanho & $\begin{array}{l}\text { Limite } \\
\text { Inferior }\end{array}$ & $\begin{array}{l}\text { Limite } \\
\text { Superior }\end{array}$ & $\mathrm{p}$-valor \\
\hline \multirow[t]{2}{*}{ IPRF (\%) } & OD & 95,88 & 100 & 5,95 & 84 & 100 & 16 & 92,96 & 98,79 & 0,397 \\
\hline & OE & 97,00 & 100 & 5,56 & 84 & 100 & 16 & 94,27 & 99,73 & \\
\hline \multirow[t]{2}{*}{ TFRB (\%) } & OD & 85,50 & 88 & 12,40 & 60 & 100 & 16 & 79,42 & 91,58 & 0,287 \\
\hline & OE & 82,25 & 80 & 10,12 & 68 & 100 & 16 & 77,29 & 87,21 & \\
\hline \multirow[t]{2}{*}{ TDNV-AL } & OD & 11,73 & 11 & 2,76 & 8 & 18 & 15 & 10,33 & 13,13 & 0,232 \\
\hline & OE & 10,00 & 10 & 3,32 & 5 & 15 & 15 & 8,32 & 11,68 & \\
\hline \multirow[t]{2}{*}{ TDNV-EDD } & OD & 20,75 & 20,5 & 1,71 & 19 & 23 & 4 & 19,08 & 22,42 & $0,002^{*}$ \\
\hline & OE & 2,75 & 2,5 & 1,71 & 1 & 5 & 4 & 1,08 & 4,42 & \\
\hline \multirow[t]{2}{*}{ TDNV-EDE } & OD & 2,50 & 2,5 & 1,29 & 1 & 4 & 4 & 1,23 & 3,77 & $0,001^{*}$ \\
\hline & OE & 20,50 & 20 & 1,73 & 19 & 23 & 4 & 18,80 & 22,20 & \\
\hline \multirow[t]{2}{*}{ SSW (\%) } & OD & 62,60 & 60 & 9,94 & 50 & 75 & 5 & 53,89 & 71,31 & 0,467 \\
\hline & OE & 57,00 & 58 & 16,49 & 35 & 80 & 5 & 42,54 & 71,46 & \\
\hline \multirow[t]{2}{*}{ PSI-40 (\%) } & OD & 96,25 & 100 & 7,44 & 80 & 100 & 8 & 91,09 & 101,41 & 0,197 \\
\hline & OE & 100,00 & 100 & 0 & 100 & 100 & 8 & $-x-$ & $-x-$ & \\
\hline \multirow[t]{2}{*}{ PSI-10 (\%) } & OD & 71,25 & 70 & 21,67 & 40 & 100 & 8 & 56,23 & 86,27 & 0,678 \\
\hline & OE & 75,00 & 80 & 20,70 & 40 & 100 & 8 & 60,65 & 89,35 & \\
\hline \multirow[t]{2}{*}{ TDD (\%) } & OD & 70,50 & 70 & 8,70 & 63 & 79 & 4 & 61,98 & 79,02 & 0,686 \\
\hline & OE & 67,50 & 67 & 9,95 & 56 & 80 & 4 & 57,75 & 77,25 & \\
\hline \multirow[t]{2}{*}{ PSI-PAL (\%) } & OD & 93,33 & 90 & 5,77 & 90 & 100 & 3 & 86,80 & 99,87 & 0,423 \\
\hline & OE & 96,67 & 100 & 5,77 & 90 & 100 & 3 & 90,13 & 103,20 & \\
\hline
\end{tabular}

Legenda: IPRF - Índice Percentual de Reconhecimento de Fala com gravação; TRFB - Teste de Fala com Ruído Branco; TDNV-AL - Teste Dicótico Não Verbal Atenção Livre; TDNV-EDD - Teste Dicótico Não Verbal - Escuta Direcionada Direta; TDNV-EDE - Teste Dicótico Não Verbal - Escuta Direcionada Esquerda; SSW - Teste Dicótico de Dissílabos Alternados; PSI-40 - Teste de Identificação de Sentenças Pediátricas (mensagem competitiva contralateral-relação fala/ruído -40dB); PSI-10 - Teste de Identificação de Sentenças Pediátricas (mensagem competitiva ipsilateral -relação fala/ruído -10dB); TDD - Teste Dicótico de Dígitos;PSI-PAL Teste de Identificação de Palavras com Ruído Branco; OD - Orelha Direita; OE - Orelha Esquerda; * p-valores considerados estatisticamente significativos perante o nível de significância adotado (Teste de Wilcoxon)

Tabela 2. Medidas descritivas das respostas corretas das orelhas direita e esquerda para os testes de avaliação comportamental do processamento auditivo na faixa etária de oito a 11 anos

\begin{tabular}{|c|c|c|c|c|c|c|c|c|c|c|}
\hline 8 a 11 anos & & Média & Mediana & $\begin{array}{l}\text { Desvio } \\
\text { Padrão }\end{array}$ & Mínimo & Máximo & Tamanho & $\begin{array}{l}\text { Limite } \\
\text { Inferior }\end{array}$ & $\begin{array}{c}\text { Limite } \\
\text { Superior }\end{array}$ & $p$-valor \\
\hline \multirow[t]{2}{*}{ IPRF (\%) } & OD & 95,00 & 94 & 4,50 & 88 & 100 & 16 & 92,79 & 97,21 & 0,580 \\
\hline & OE & 95,50 & 96 & 3,83 & 88 & 100 & 16 & 93,62 & 97,38 & \\
\hline \multirow[t]{2}{*}{ TFRB (\%) } & OD & 86,25 & 88 & 10,93 & 64 & 100 & 16 & 80,90 & 91,60 & 0,218 \\
\hline & OE & 88,50 & 94 & 12,21 & 64 & 100 & 16 & 82,52 & 94,48 & \\
\hline \multirow[t]{2}{*}{ TDNV-AL } & OD & 10,88 & 11 & 2,06 & 8 & 15 & 16 & 9,86 & 11,89 & $0,113 \#$ \\
\hline & OE & 12,69 & 13 & 2,33 & 9 & 16 & 16 & 11,55 & 13,83 & \\
\hline \multirow[t]{2}{*}{ TDNV-EDD } & OD & 20,67 & 22 & 4,81 & 7 & 24 & 15 & 18,23 & 23,10 & $<0,001^{\star}$ \\
\hline & OE & 2,87 & 1 & 4,78 & 0 & 17 & 15 & 0,45 & 5,29 & \\
\hline \multirow[t]{2}{*}{ TDNV-EDE } & OD & 1,73 & 1 & 3,10 & 0 & 12 & 15 & 0,16 & 3,30 & $<0,001^{\star}$ \\
\hline & OE & 21,13 & 23 & 3,42 & 12 & 24 & 15 & 19,40 & 22,86 & \\
\hline \multirow[t]{2}{*}{ SSW (\%) } & OD & 82,67 & 85 & 12,25 & 58 & 100 & 15 & 76,47 & 88,86 & $0,083^{*}$ \\
\hline & OE & 74,27 & 80 & 21,74 & 28 & 100 & 15 & 63,26 & 85,27 & \\
\hline \multirow[t]{2}{*}{ PSI-40 (\%) } & OD & 100,00 & 100 & 0 & 100 & 100 & 6 & $-x-$ & $-x-$ & 1,000 \\
\hline & OE & 100,00 & 100 & 0 & 100 & 100 & 6 & $-x-$ & $-x-$ & \\
\hline \multirow[t]{2}{*}{ PSI-10 (\%) } & OD & 83,33 & 85 & 13,66 & 60 & 100 & 6 & 72,40 & 94,27 & 0,679 \\
\hline & OE & 80,00 & 85 & 17,89 & 50 & 100 & 6 & 65,69 & 94,31 & \\
\hline
\end{tabular}

Legenda: IPRF - Índice Percentual de Reconhecimento de Fala com gravação; TRFB - Teste de Fala com Ruído Branco; TDNV-AL - Teste Dicótico Não Verbal Atenção Livre; TDNV-EDD - Teste Dicótico Não Verbal - Escuta Direcionada Direta; TDNV-EDE - Teste Dicótico Não Verbal - Escuta Direcionada Esquerda; SSW - Teste Dicótico de Dissílabos Alternados; PSI-40 - Teste de Identificação de Sentenças Pediátricas (mensagem competitiva contralateral-relação fala/ruído -40dB); PSI-10 - Teste de Identificação de Sentenças Pediátricas (mensagem competitiva ipsilateral -relação fala/ruído -10dB); OD - Orelha Direita; OE - Orelha Esquerda; * p-valores considerados estatisticamente significativos perante o nível de significância adotado (Teste de Wilcoxon); \# p-valores que por estarem próximos do limite de aceitação, são considerados que tendem a ser significativos (Teste de Wilcoxon) 
Tabela 3. Medidas descritivas das respostas corretas das orelhas direita e esquerda para os testes de avaliação comportamental do processamento auditivo na faixa etária de 12 a 34 anos

\begin{tabular}{|c|c|c|c|c|c|c|c|c|c|c|}
\hline$>12$ anos & & Média & Mediana & $\begin{array}{l}\text { Desvio } \\
\text { Padrão }\end{array}$ & Mín & Máx & Tamanho & $\begin{array}{l}\text { Limite } \\
\text { Inferior }\end{array}$ & $\begin{array}{l}\text { Limite } \\
\text { Superior }\end{array}$ & p-valor \\
\hline \multirow[t]{2}{*}{ IPRF (\%) } & OD & 95,50 & 96 & 3,60 & 88 & 100 & 24 & 94,06 & 96,94 & 0,524 \\
\hline & $\mathrm{OE}$ & 96,00 & 96 & 3,54 & 88 & 100 & 24 & 94,58 & 97,42 & \\
\hline \multirow[t]{2}{*}{ TFRB (\%) } & OD & 93,67 & 96 & 5,65 & 84 & 100 & 24 & 91,41 & 95,93 & $0,121 \#$ \\
\hline & OE & 91,83 & 92 & 6,07 & 80 & 100 & 24 & 89,41 & 94,26 & \\
\hline \multirow[t]{2}{*}{ TDNV-AL } & OD & 12,22 & 12 & 1,98 & 8 & 16 & 23 & 11,41 & 13,03 & 0,506 \\
\hline & OE & 11,65 & 12 & 2,06 & 8 & 16 & 23 & 10,81 & 12,49 & \\
\hline \multirow[t]{2}{*}{ TDNV-EDD } & OD & 21,55 & 23 & 3,40 & 12 & 24 & 22 & 20,12 & 22,97 & $<0,001^{*}$ \\
\hline & OE & 1,95 & 1 & 3,29 & 0 & 12 & 22 & 0,58 & 3,33 & \\
\hline \multirow[t]{2}{*}{ TDNV-EDE } & OD & 1,05 & 0 & 2,19 & 0 & 10 & 22 & 0,13 & 1,96 & $<0,001^{*}$ \\
\hline & OE & 21,82 & 23 & 3,59 & 11 & 24 & 22 & 20,32 & 23,32 & \\
\hline \multirow[t]{2}{*}{ TPF (\%) } & OD & 56,57 & 56 & 28,84 & 10 & 90 & 7 & 35,20 & 77,94 & 0,604 \\
\hline & OE & 54,86 & 60 & 28,48 & 17 & 94 & 7 & 33,76 & 75,96 & \\
\hline \multirow[t]{2}{*}{ TPD (\%) } & OD & 69,67 & 69,5 & 19,95 & 33 & 97 & 12 & 58,38 & 80,96 & 0,683 \\
\hline & $\mathrm{OE}$ & 68,25 & 70 & 20,42 & 33 & 93 & 12 & 56,69 & 79,81 & \\
\hline \multirow[t]{2}{*}{ SSW (\%) } & OD & 90,79 & 94 & 7,49 & 72 & 100 & 24 & 87,79 & 93,79 & $0,142 \#$ \\
\hline & OE & 87,46 & 90 & 7,48 & 73 & 98 & 24 & 84,46 & 90,45 & \\
\hline \multirow[t]{2}{*}{ SSI-40 (\%) } & OD & 100,00 & 100 & 0 & 100 & 100 & 10 & $-x-$ & $-x-$ & 1,000 \\
\hline & $\mathrm{OE}$ & 100,00 & 100 & 0 & 100 & 100 & 10 & $-x-$ & $-x-$ & \\
\hline \multirow[t]{2}{*}{ SSI-10 (\%) } & OD & 74,00 & 70 & 13,50 & 60 & 100 & 10 & 65,63 & 82,37 & 0,309 \\
\hline & $\mathrm{OE}$ & 78,00 & 75 & 14,76 & 60 & 100 & 10 & 68,85 & 87,15 & \\
\hline \multirow[t]{2}{*}{ TDCV-AL } & OD & 10,40 & 11 & 1,71 & 8 & 12 & 10 & 9,34 & 11,46 & $0,030^{*}$ \\
\hline & OE & 8,20 & 8,5 & 1,99 & 5 & 11 & 10 & 6,97 & 9,43 & \\
\hline \multirow[t]{2}{*}{ TDCV-EDD } & OD & 11,30 & 11 & 3,47 & 5 & 17 & 10 & 9,15 & 13,45 & $0,111 \#$ \\
\hline & $\mathrm{OE}$ & 7,70 & 7,5 & 3,13 & 2 & 13 & 10 & 5,76 & 9,64 & \\
\hline \multirow[t]{2}{*}{ TDCV-EDE } & OD & 9,30 & 9 & 2,31 & 6 & 14 & 10 & 7,87 & 10,73 & 0,578 \\
\hline & $\mathrm{OE}$ & 8,40 & 9 & 3,27 & 2 & 13 & 10 & 6,37 & 10,43 & \\
\hline
\end{tabular}

Legenda: Mín - Mínimo; Máx - Máximo; IPRF - Índice Percentual de Reconhecimento de Fala com gravação; TRFB - Teste de Fala com Ruído Branco; TDNV-AL - Teste Dicótico Não Verbal - Atenção Livre; TDNV-EDD - Teste Dicótico Não Verbal - Escuta Direcionada Direta; TDNV-EDE - Teste Dicótico Não Verbal - Escuta Direcionada Esquerda; TPF - Teste de Reconhecimento de Padrão de Freqüência; TPD - Teste de Reconhecimento de Padrão de Duração; SSW - Teste Dicótico de Dissílabos Alternados; SSI-40 - Teste de Identificação de Sentenças Sintéticas (mensagem competitiva contralateral-relação fala/ruído -40dB); SSI-10 - Teste de Identificação de Sentenças Sintéticas (mensagem competitiva ipsilateral-relação fala/ruído -10dB); TDCV-AL - Teste Dicótico Consoante Vogal - Atenção Livre; TDCVEDD - Teste Dicótico Consoante Vogal - Escuta Direcionada à Direita; TDCV-EDE - Teste Dicótico Consoante Vogal - Escuta Direcionada à Esquerda; OD - Orelha Direita; OE - Orelha Esquerda; * p-valores considerados estatisticamente significativos perante o nível de significância adotado (Teste de Wilcoxon); \# p-valores que por estarem próximos do limite de aceitação, são considerados que tendem a ser significativos (Teste de Wilcoxon)

Tabela 4. Medidas descritivas dos testes de avaliação comportamental do processamento auditivo na faixa etária de quatro a sete anos e os diferentes graus de gravidade da gagueira

\begin{tabular}{|c|c|c|c|c|c|c|c|c|c|c|}
\hline \multirow[t]{2}{*}{4 a 7 anos } & & Média & Mediana & Desvio & Min & Máx & Tamanho & Limite & Limite & $p$-valor \\
\hline & & & & Padrão & & & & Inferior & Superior & \\
\hline \multirow[t]{3}{*}{ IPRF (\%) } & leve & 97,40 & 100 & 5,07 & 84 & 100 & 20 & 95,18 & 99,62 & 0,247 \\
\hline & moderado & 94,00 & 96 & 6,57 & 84 & 100 & 6 & 88,74 & 99,26 & \\
\hline & severo & 95,67 & 100 & 6,98 & 84 & 100 & 6 & 90,08 & 101,25 & \\
\hline \multirow[t]{3}{*}{ TFRB (\%) } & leve & 86,60 & 88 & 11,03 & 70 & 100 & 20 & 81,76 & 91,44 & 0,183 \\
\hline & moderado & 82,67 & 84 & 8,64 & 68 & 92 & 6 & 75,75 & 89,58 & \\
\hline & severo & 76,00 & 75 & 11,93 & 60 & 90 & 6 & 66,45 & 85,55 & \\
\hline \multirow[t]{3}{*}{ TDNV-AL } & leve & 10,89 & 10,5 & 3,32 & 5 & 15 & 18 & 9,35 & 12,42 & 0,988 \\
\hline & moderado & 11,00 & 10,5 & 4,20 & 5 & 18 & 6 & 7,64 & 14,36 & \\
\hline & severo & 10,67 & 10,5 & 1,21 & 9 & 12 & 6 & 9,70 & 11,64 & \\
\hline \multirow[t]{3}{*}{ SSW (\%) } & leve & 68,75 & 72,5 & 13,15 & 50 & 80 & 4 & 55,86 & 81,64 & 0,189 \\
\hline & moderado & 60,00 & 60 & 2,83 & 58 & 62 & 2 & 56,08 & 63,92 & \\
\hline & severo & 50,75 & 54 & 11,35 & 35 & 60 & 4 & 39,62 & 61,88 & \\
\hline \multirow[t]{3}{*}{ PSI-40 (\%) } & leve & 97,50 & 100 & 6,22 & 80 & 100 & 12 & 93,98 & 101,02 & 0,701 \\
\hline & moderado & 100,00 & 100 & 0,00 & 100 & 100 & 2 & $-x-$ & $-x-$ & \\
\hline & severo & 100,00 & 100 & 0,00 & 100 & 100 & 2 & $-x-$ & $-x-$ & \\
\hline \multirow[t]{3}{*}{ PSI-10 (\%) } & leve & 78,33 & 80 & 18,01 & 40 & 100 & 12 & 68,15 & 88,52 & $0,141 \#$ \\
\hline & moderado & 45,00 & 45 & 7,07 & 40 & 50 & 2 & 35,20 & 54,80 & \\
\hline & severo & 70,00 & 70 & 28,28 & 50 & 90 & 2 & 30,80 & 109,20 & \\
\hline \multirow[t]{2}{*}{ PSI-PAL (\%) } & leve & 92,50 & 90 & 5,00 & 90 & 100 & 4 & 87,60 & 97,40 & $0,114 \#$ \\
\hline & $\begin{array}{l}\text { moderado } \\
\text { severo }\end{array}$ & 100,00 & 100 & 0,00 & 100 & 100 & 2 & $-x-$ & $-x-$ & \\
\hline
\end{tabular}

Legenda: Mín - Mínimo; Máx - Máximo; IPRF - Índice Percentual de Reconhecimento de Fala com gravação; TRFB - Teste de Fala com Ruído Branco; TDNV-AL - Teste Dicótico Não Verbal - Atenção Livre; SSW - Teste Dicótico de Dissílabos Alternados; PSI-40 - Teste de Identificação de Sentenças Pediátricas (mensagem competitiva contralateral-relação fala/ruído -40dB); PSI-10 - Teste de Identificação de Sentenças Pediátricas (mensagem competitiva ipsilateral-relação fala/ruído -10dB); PSI-PAL - Teste de Identificação de Palavras com Ruído Branco; \# p-valores que por estarem próximos do limite de aceitação, são considerados que tendem a ser significativos (Teste Kruskal-Wallis) 
Tabela 5. Medidas descritivas dos testes de avaliação comportamental do processamento auditivo na faixa etária de oito a 11 anos e os diferentes graus de gravidade da gagueira.

\begin{tabular}{|c|c|c|c|c|c|c|c|c|c|c|}
\hline 8 a 11 anos & & Média & Mediana & $\begin{array}{l}\text { Desvio } \\
\text { Padrão }\end{array}$ & Mínimo & Máximo & Tamanho & $\begin{array}{l}\text { Limite } \\
\text { Inferior }\end{array}$ & $\begin{array}{c}\text { Limite } \\
\text { Superior }\end{array}$ & $p$-valor \\
\hline \multirow{4}{*}{ IPRF (\%) } & muito leve & 9467 & 94 & & 88 & 100 & 6 & & & 0408 \\
\hline & leve & 96.80 & 100 & 4,54 & 88 & 100 & 10 & 93,99 & 99,61 & \\
\hline & moderado & 94,86 & 94 & 3,30 & 92 & 100 & 14 & 93,13 & 96,59 & \\
\hline & severo & 92,00 & 92 & 5,66 & 88 & 96 & 2 & 84,16 & 99,84 & \\
\hline \multirow[t]{4}{*}{ TFRB (\%) } & muito leve & 94,67 & 96 & 6,53 & 84 & 100 & 6 & 89,44 & 99,89 & 0,205 \\
\hline & leve & 83,20 & 88 & 13,04 & 64 & 96 & 10 & 75,12 & 91,28 & \\
\hline & moderado & 87,71 & 92 & 11,68 & 64 & 100 & 14 & 81,59 & 93,83 & \\
\hline & severo & 84,00 & 84 & 5,66 & 80 & 88 & 2 & 76,16 & 91,84 & \\
\hline \multirow[t]{4}{*}{ TDNV-AL } & muito leve & 12,00 & 12 & 3,41 & 8 & 16 & 6 & 9,27 & 14,73 & 0,997 \\
\hline & leve & 11,70 & 12 & 1,64 & 9 & 15 & 10 & 10,69 & 12,71 & \\
\hline & moderado & 11,79 & 11,5 & 2,22 & 9 & 15 & 14 & 10,62 & 12,95 & \\
\hline & severo & 11,50 & 11,5 & 4,95 & 8 & 15 & 2 & 4,64 & 18,36 & \\
\hline \multirow[t]{4}{*}{ TDNV-EDD } & muito leve & 11,50 & 11 & 11,36 & 0 & 24 & 4 & 0,37 & 22,63 & 1,000 \\
\hline & leve & 11,90 & 12 & 11,61 & 0 & 24 & 10 & 4,70 & 19,10 & \\
\hline & moderado & 11,71 & 12 & 9,33 & 0 & 24 & 14 & 6,83 & 16,60 & \\
\hline & severo & 12,00 & 12 & 16,97 & 0 & 24 & 2 & $-11,52$ & 35,52 & \\
\hline \multirow[t]{4}{*}{ TDNV-EDE } & muito leve & 11,00 & 10,5 & 9,90 & 2 & 21 & 4 & 1,30 & 20,70 & 0,995 \\
\hline & leve & 11,40 & 10,5 & 11,47 & 0 & 24 & 10 & 4,29 & 18,51 & \\
\hline & moderado & 11,50 & 12 & 10,38 & 0 & 24 & 14 & 6,07 & 16,93 & \\
\hline & severo & 12,00 & 12 & 15,56 & 1 & 23 & 2 & $-9,56$ & 33,56 & \\
\hline \multirow[t]{4}{*}{ SSW (\%) } & muito leve & 83,75 & 82,5 & 8,54 & 75 & 95 & 4 & 75,38 & 92,12 & $0,122 \#$ \\
\hline & leve & 87,70 & 90 & 9,81 & 70 & 100 & 10 & 81,62 & 93,78 & \\
\hline & moderado & 75,07 & 77,5 & 18,71 & 28 & 97 & 14 & 65,27 & 84,87 & \\
\hline & severo & 45,50 & 45,5 & 17,68 & 33 & 58 & 2 & 21,00 & 70,00 & \\
\hline \multirow[t]{3}{*}{ PSI-40 (\%) } & muito leve & 100,00 & 100 & 0,00 & 100 & 100 & 2 & $-x-$ & $-x-$ & 1,000 \\
\hline & leve & 100,00 & 100 & 0,00 & 100 & 100 & 6 & $-x-$ & $-x-$ & \\
\hline & $\begin{array}{l}\text { moderado } \\
\text { severo }\end{array}$ & 100,00 & 100 & 0,00 & 100 & 100 & 4 & $-x-$ & $-x-$ & \\
\hline \multirow[t]{3}{*}{ PSI-10 (\%) } & muito leve & 90,00 & 90 & 0,00 & 90 & 90 & 2 & $-x-$ & $-x-$ & $0,131 \#$ \\
\hline & leve & 88,33 & 90 & 11,69 & 70 & 100 & 6 & 78,98 & 97,69 & \\
\hline & $\begin{array}{l}\text { moderado } \\
\text { severo }\end{array}$ & 67,50 & 70 & 15,00 & 50 & 80 & 4 & 52,80 & 82,20 & \\
\hline
\end{tabular}

Legenda: IPRF - Índice Percentual de Reconhecimento de Fala com gravação; TRFB - Teste de Fala com Ruído Branco; TDNV-AL - Teste Dicótico Não Verbal Atenção Livre; TDNV-EDD - Teste Dicótico Não Verbal - Escuta Direcionada Direta; TDNV-EDE - Teste Dicótico Não Verbal - Escuta Direcionada Esquerda; SSW - Teste Dicótico de Dissílabos Alternados; PSI-40 - Teste de Identificação de Sentenças Pediátricas (mensagem competitiva contralateral-relação fala/ruído -40dB); PSI-10 - Teste de Identificação de Sentenças Pediátricas (mensagem competitiva ipsilateral-relação fala/ruído -10dB); \# p-valores que por estarem próximos do limite de aceitação, são considerados que tendem a ser significativos (Teste Kruskal-Wallis)

Quadro 2. P-valores entre o grau de gravidade da gagueira e as diferentes faixas etárias

\begin{tabular}{|lccc|}
\hline $\begin{array}{l}\text { Grau de gravidade da } \\
\text { gagueira }\end{array}$ & Faixa etária & 4 a 7 anos & 8 a 11 anos \\
\hline Muito leve & 8 a 11 anos & $0,069^{*}$ & \\
Leve & $>12$ anos & $0,085^{*}$ & 0,865 \\
& 8 a 11 anos & $0,077^{*}$ & \\
Moderado & $>12$ anos & 0,436 & 0,240 \\
& 8 a 11 anos & $0,127 \#$ & \\
Severo & $>12$ anos & 0,872 & $0,121 \#$ \\
& 8 a 11 anos & 0,285 & \\
& $>12$ anos & 0,588 & 0,519 \\
\hline
\end{tabular}

Legenda: Teste de Igualdade de Duas Proporções

* $\mathrm{p}$-valores considerados estatisticamente significativos perante o nível de significância adotado.

\# p-valores que por estarem próximos do limite de aceitação, são considerados que tendem a ser significativos. resposta. Nos locais onde não foi possível o cálculo de intervalo de confiança, pois não havia desvio padrão (todos os indivíduos possuem o mesmo valor), foi utilizado o símbolo “- $\mathrm{x}$-". Nas medidas onde não havia valores para os níveis de gravidade de gagueira, o espaço foi deixado em branco.

\section{DISCUSSÃO}

Para analisar os resultados deste estudo os dividimos em duas partes:

Parte 1. Comparação do número de respostas corretas das orelhas direita e esquerda para cada teste do processamento auditivo, segundo a variável faixa etária

Nesta pesquisa, encontramos alteração do processamento auditivo em 52 indivíduos (92,85\%). Pesquisas demonstram que como um grupo, os gagos apresentam evidências de distúrbio do processamento auditivo ${ }^{(17)}$. Os achados observados 
Tabela 6. Medidas descritivas dos testes de avaliação comportamental do processamento auditivo na faixa etária de 12 a 34 anos e os diferentes graus de gravidade da gagueira

\begin{tabular}{|c|c|c|c|c|c|c|c|c|c|c|}
\hline$>12$ anos & & Média & Mediana & $\begin{array}{l}\text { Desvio } \\
\text { Padrão }\end{array}$ & Mínimo & Máximo & Tamanho & $\begin{array}{l}\text { Limite } \\
\text { Inferior }\end{array}$ & $\begin{array}{l}\text { Limite } \\
\text { Superior }\end{array}$ & $p$-valor \\
\hline \multirow[t]{5}{*}{ IPRF (\%) } & muito leve & 98,00 & 100 & 3,02 & 92 & 100 & 8 & 95,90 & 100,10 & $0,102 \#$ \\
\hline & leve & 96,00 & 96 & 3,54 & 88 & 100 & 24 & 94,58 & 97,42 & \\
\hline & moderado & 95,20 & 96 & 3,16 & 92 & 100 & 10 & 93,24 & 97,16 & \\
\hline & severo & 93,00 & 94 & 3,83 & 88 & 96 & 4 & 89,25 & 96,75 & \\
\hline & muito severo & 92,00 & 92 & 0,00 & 92 & 92 & 2 & $-x-$ & $-x-$ & \\
\hline \multirow[t]{5}{*}{ TFRB (\%) } & muito leve & 93,00 & 92 & 2,83 & 88 & 96 & 8 & 91,04 & 94,96 & $0,135 \#$ \\
\hline & leve & 94,50 & 96 & 5,63 & 84 & 100 & 24 & 92,25 & 96,75 & \\
\hline & moderado & 90,40 & 90 & 7,35 & 80 & 100 & 10 & 85,84 & 94,96 & \\
\hline & severo & 92,00 & 92 & 4,62 & 88 & 96 & 4 & 87,47 & 96,53 & \\
\hline & muito severo & 84,00 & 84 & 0,00 & 84 & 84 & 2 & $-x-$ & $-x-$ & \\
\hline \multirow[t]{5}{*}{ TDNV-AL } & muito leve & 11,88 & 12 & 2,17 & 8 & 16 & 8 & 10,37 & 13,38 & 0,998 \\
\hline & leve & 12,00 & 12 & 2,14 & 8 & 16 & 22 & 11,11 & 12,89 & \\
\hline & moderado & 11,80 & 12 & 1,14 & 9 & 13 & 10 & 11,10 & 12,50 & \\
\hline & severo & 12,00 & 12 & 3,65 & 8 & 16 & 4 & 8,42 & 15,58 & \\
\hline & muito severo & 12,00 & 12 & 1,41 & 11 & 13 & 2 & 10,04 & 13,96 & \\
\hline \multirow[t]{5}{*}{ TDNV-EDD } & muito leve & 11,75 & 11 & 11,59 & 0 & 24 & 8 & 3,72 & 19,78 & 1,000 \\
\hline & leve & 11,95 & 12 & 10,43 & 0 & 24 & 20 & 7,38 & 16,52 & \\
\hline & moderado & 11,20 & 10,5 & 10,50 & 0 & 24 & 10 & 4,69 & 17,71 & \\
\hline & severo & 12,00 & 12 & 11,60 & 1 & 23 & 4 & 0,63 & 23,37 & \\
\hline & muito severo & 12,00 & 12 & 16,97 & 0 & 24 & 2 & $-11,52$ & 35,52 & \\
\hline \multirow[t]{5}{*}{ TDNV-EDE } & muito leve & 12,00 & 12 & 12,33 & 0 & 24 & 8 & 3,46 & 20,54 & 0,992 \\
\hline & leve & 11,20 & 10,5 & 11,06 & 0 & 24 & 20 & 6,35 & 16,05 & \\
\hline & moderado & 11,20 & 11,5 & 10,39 & 0 & 23 & 10 & 4,76 & 17,64 & \\
\hline & severo & 11,75 & 12 & 12,45 & 0 & 23 & 4 & $-0,45$ & 23,95 & \\
\hline & muito severo & 12,00 & 12 & 16,97 & 0 & 24 & 2 & $-11,52$ & 35,52 & \\
\hline \multirow[t]{4}{*}{ TPF (\%) } & muito leve & 28,00 & 28 & 7,07 & 23 & 33 & 2 & 18,20 & 37,80 & 0,299 \\
\hline & leve & 55,13 & 60 & 30,78 & 10 & 94 & 8 & 33,79 & 76,46 & \\
\hline & moderado & 70,75 & 68,5 & 15,73 & 56 & 90 & 4 & 55,33 & 86,17 & \\
\hline & $\begin{array}{l}\text { severo } \\
\text { muito severo }\end{array}$ & & & & & & & & & \\
\hline \multirow[t]{5}{*}{ TPD (\%) } & leve & 70,60 & 73 & 20,60 & 33 & 93 & 10 & 57,83 & 83,37 & 0,181 \\
\hline & muito leve & 85,00 & 86,5 & 6,27 & 77 & 90 & 4 & 78,85 & 91,15 & \\
\hline & moderado & 73,25 & 71,5 & 20,37 & 53 & 97 & 4 & 53,29 & 93,21 & \\
\hline & severo & 48,25 & 50 & 13,87 & 33 & 60 & 4 & 34,66 & 61,84 & \\
\hline & muito severo & 61,50 & 61,5 & 16,26 & 50 & 73 & 2 & 38,96 & 84,04 & \\
\hline \multirow[t]{5}{*}{ SSW (\%) } & muito leve & 91,13 & 92,5 & 8,46 & 72 & 98 & 8 & 85,26 & 96,99 & 0,798 \\
\hline & leve & 88,08 & 89 & 7,68 & 75 & 100 & 24 & 85,01 & 91,16 & \\
\hline & moderado & 90,10 & 93,5 & 7,71 & 73 & 97 & 10 & 85,32 & 94,88 & \\
\hline & severo & 88,50 & 92 & 9,11 & 75 & 95 & 4 & 79,57 & 97,43 & \\
\hline & muito severo & 90,00 & 90 & 0,00 & 90 & 90 & 2 & $-x-$ & $-x-$ & \\
\hline \multirow[t]{5}{*}{ SSI-40 (\%) } & muito leve & 100,00 & 100 & 0,00 & 100 & 100 & 4 & $-x-$ & $-x-$ & 1,000 \\
\hline & leve & 100,00 & 100 & 0,00 & 100 & 100 & 6 & $-x-$ & $-x-$ & \\
\hline & moderado & 100,00 & 100 & 0,00 & 100 & 100 & 4 & $-x-$ & $-x-$ & \\
\hline & severo & 100,00 & 100 & 0,00 & 100 & 100 & 4 & $-x-$ & $-x-$ & \\
\hline & muito severo & 100,00 & 100 & 0,00 & 100 & 100 & 2 & $-x-$ & $-x-$ & \\
\hline \multirow[t]{5}{*}{ SSI-10 (\%) } & muito leve & 75,00 & 80 & 10,00 & 60 & 80 & 4 & 65,20 & 84,80 & 0,719 \\
\hline & leve & 75,00 & 70 & 16,43 & 60 & 100 & 6 & 61,85 & 88,15 & \\
\hline & moderado & 80,00 & 80 & 18,26 & 60 & 100 & 4 & 62,11 & 97,89 & \\
\hline & severo & 80,00 & 75 & 14,14 & 70 & 100 & 4 & 66,14 & 93,86 & \\
\hline & muito severo & 65,00 & 65 & 7,07 & 60 & 70 & 2 & 55,20 & 74,80 & \\
\hline \multirow[t]{4}{*}{ TDCV-AL } & muito leve & 9,33 & 10 & 2,88 & 5 & 12 & 6 & 7,03 & 11,63 & 0,904 \\
\hline & leve & 9,33 & 9 & 1,86 & 7 & 12 & 6 & 7,84 & 10,82 & \\
\hline & moderado & 9,25 & 9,5 & 2,75 & 6 & 12 & 4 & 6,55 & 11,95 & \\
\hline & $\begin{array}{l}\text { severo } \\
\text { muito severo }\end{array}$ & 9,25 & 9 & 1,26 & 8 & 11 & 4 & 8,02 & 10,48 & \\
\hline
\end{tabular}


Tabela 6. continuação

\begin{tabular}{|c|c|c|c|c|c|c|c|c|c|c|}
\hline \multirow[t]{2}{*}{$>12$ anos } & & \multirow[t]{2}{*}{ Média } & \multirow[t]{2}{*}{ Mediana } & \multirow{2}{*}{$\begin{array}{l}\text { Desvio } \\
\text { Padrão }\end{array}$} & \multirow[t]{2}{*}{ Mínimo } & \multirow[t]{2}{*}{ Máximo } & \multirow[t]{2}{*}{ Tamanho } & \multirow{2}{*}{$\begin{array}{l}\text { Limite } \\
\text { Inferior }\end{array}$} & \multirow{2}{*}{$\begin{array}{c}\text { Limite } \\
\text { Superior }\end{array}$} & \multirow[t]{2}{*}{$p$-valor } \\
\hline & & & & & & & & & & \\
\hline \multirow[t]{5}{*}{ TDCV-EDD } & muito leve & 9,67 & 10,5 & 4,97 & 2 & 17 & 6 & 5,69 & 13,64 & 0,975 \\
\hline & leve & 10,00 & 9,5 & 4,00 & 5 & 15 & 6 & 6,80 & 13,20 & \\
\hline & moderado & 9,00 & 9 & 2,58 & 6 & 12 & 4 & 6,47 & 11,53 & \\
\hline & severo & 9,00 & 9 & 3,37 & 5 & 13 & 4 & 5,70 & 12,30 & \\
\hline & muito severo & & & & & & & & & \\
\hline \multirow[t]{4}{*}{ TDCV-EDE } & muito leve & 8,17 & 9 & 3,19 & 2 & 11 & 6 & 5,62 & 10,72 & 0,677 \\
\hline & leve & 8,33 & 7,5 & 3,39 & 5 & 14 & 6 & 5,62 & 11,04 & \\
\hline & moderado & 9,00 & 9 & 1,63 & 7 & 11 & 4 & 7,40 & 10,60 & \\
\hline & $\begin{array}{l}\text { severo } \\
\text { muito severo }\end{array}$ & 10,50 & 10,5 & 2,38 & 8 & 13 & 4 & 8,17 & 12,83 & \\
\hline
\end{tabular}

Legenda: IPRF - Índice Percentual de Reconhecimento de Fala com gravação; TRFB - Teste de Fala com Ruído Branco; TDNV-AL - Teste Dicótico Não Verbal - Atenção Livre; TDNV-EDD - Teste Dicótico Não Verbal - Escuta Direcionada Direta; TDNV-EDE - Teste Dicótico Não Verbal - Escuta Direcionada Esquerda; TPF - Teste de Reconhecimento de Padrão de Freqüência; TPD - Teste de Reconhecimento de Padrão de Duração; SSW - Teste Dicótico de Dissílabos Alternados; SSI-40 - Teste de Identificação de Sentenças Sintéticas (mensagem competitiva contralateral-relação fala/ruído -40dB); SSI-10 - Teste de Identificação de Sentenças Sintéticas (mensagem competitiva ipsilateral-relação fala/ ruído -10dB); TDCV-AL - Teste Dicótico Consoante Vogal - Atenção Livre; TDCV-EDD - Teste Dicótico Consoante Vogal - Escuta Direcionada à Direita; TDCV-EDE - Teste Dicótico Consoante Vogal - Escuta Direcionada à Esquerda; \# p-valores que por estarem próximos do limite de aceitação, são considerados que tendem a ser significativos (Teste Kruskal-Wallis)

no presente estudo foram semelhantes aos de outros estudos encontrados na literatura, os quais encontraram alteração na avaliação comportamental do PA em mais de $90 \%$ dos indi-

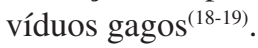

Os hemisférios cerebrais são organizados assimetricamente, sendo que o hemisfério cerebral esquerdo é mais especializado para a linguagem e o direito, mais envolvido com os componentes visuais e espaciais em indivíduos destros ${ }^{(20)}$.

Pesquisas realizadas valorizam a teoria de que os indivíduos gagos não possuem dominância hemisférica para a fala, possuem dominância hemisférica invertida ou dominância hemisférica bilateral ${ }^{(11)}$. Estudos realizados com teste de escuta dicótica não revelaram diferenças nas respostas obtidas entre grupos de gagos adultos e o grupo controle ${ }^{(21)}$.

No presente estudo, para todas as faixas etárias houve diferença estatisticamente significante entre as orelhas direita e esquerda, nas etapas de atenção direcionada do teste dicótico não verbal (TDNV-EDD e TDNV-EDE). Este resultado encontra-se dentro dos padrões de normalidade, uma vez que nestas etapas, o paciente é solicitado a apontar o estímulo apresentado primeiro à direita (atenção direita) e, posteriormente, à esquerda (atenção esquerda).

As etapas de escuta direcionada do teste dicótico não verbal sugerem integridade nos centros de associações do hemisfério esquerdo e/ou corpo caloso, quando o estímulo é apresentado à orelha direita e no hemisfério direito, quando o estímulo é apresentado à orelha esquerda ${ }^{(22)}$.

$\mathrm{Na}$ avaliação quantitativa do SSW (\%), houve diferença média estatisticamente significante entre as orelhas direita e esquerda, sendo o desempenho da orelha direita superior ao desempenho da orelha esquerda na faixa etária de oito a 11 anos. Nesta faixa etária, especialmente em crianças de oito anos, a discrepância entre os resultados da orelha direita e orelha esquerda, favorecendo a orelha direita, ainda pode ser justificada por efeito da neuromaturação. Até os oito anos, ainda consideramos limites de normalidade separados para cada orelha; estes resultados corroboraram com um estudo que encontrou interferência do desenvolvimento e do processo de alfabetização em crianças de cinco a nove anos incompletos, quando avaliou a habilidade auditiva de figura-fundo para palavras dissílabas em tarefa dicótica $(\mathrm{SSW})^{(23)}$.

Para a faixa etária de 12 a 34 anos, houve diferença média estatisticamente significante entre as orelhas direita e esquerda, na etapa de atenção livre do teste dicótico consoante vogal (TDCV-AL). Estes resultados são semelhantes aos descritos na literatura ${ }^{(24-25)}$.

A partir do teste dicótico consoante vogal na etapa de atenção livre, podemos inferir a respeito da dominância hemisférica para sons de fala, ou seja, se há assimetria perceptual para estímulos lingüísticos avaliados por meio da atenção livre ${ }^{(16)}$.

As diferenças entre as orelhas direita e esquerda nos testes verbais de escuta dicótica refletem as diferenças funcionais entre os hemisférios cerebrais e o fato de cada orelha ter conecção mais forte com o hemisfério contralateral ${ }^{(26-27)}$.

A vantagem de respostas corretas da orelha direita nos testes de escuta dicótica poderia ser interpretada como refletindo o hemisfério cerebral esquerdo, especializado para o processamento de fala e linguagem ${ }^{(27-28)}$.

Os resultados encontrados neste estudo que indicam a vantagem da orelha direita nos testes de escuta dicótica, corroboram os descritos na literatura ${ }^{(9,29)}$.

Parte 2: Comparação do número de respostas corretas nos testes do processamento auditivo nas diferentes faixas etárias, segundo a variável grau de gravidade da gagueira

Comentamos nesta seção a comparação do número de respostas corretas nos testes do processamento auditivo, nas diferentes faixas etárias, segundo a variável grau de gravidade da gagueira.

De acordo com os resultados sobre o grau de gravidade da 
gagueira, houve uma prevalência do grau leve nas faixas etárias de quatro a sete anos (62,5\%) e > 12 anos (50,0\%); estes dados corroboram com as pesquisas que encontraram maior número de indivíduos com gagueira de grau leve ${ }^{(18)}$.

Para a análise dos testes do processamento auditivo segundo a variável grau de gravidade da gagueira, verificamos que em nenhum dos testes do processamento auditivo, existe diferença média estatisticamente significante entre os graus de gravidade da gagueira, nas diferentes faixas etárias. Estes resultados corroboram com os descritos na literatura ${ }^{(19)}$, os quais não associam o grau de gravidade da gagueira com alteração nos testes comportamentais especiais do processamento.

Estudos realizados demonstram que indivíduos gagos possuem diferenças de desempenho, quando comparados a indivíduos não gagos no que se refere ao processamento da função de fala e processamento auditivo. As alterações encontradas, dentro das avaliações comportamentais do processamento auditivo, sugerem uma disfunção neurológica como uma das causas subjacentes para a disfluência ${ }^{(30)}$.

Verificamos que a alteração do PA foi bastante freqüente na população estudada (92\%); desta forma, acreditamos que a avaliação do PA deva fazer parte do processo diagnóstico da gagueira e que o processo terapêutico de indivíduos com gagueira e alteração do PA inclua, tanto estratégias para o treinamento das habilidades de fala (respiração, fonação, articulação e prosódia), quanto para o treinamento das habilidades auditivas subjacentes aos processos gnósicos auditivos freqüentemente prejudicados.

\section{CONCLUSÃO}

Com base nos resultados da avaliação comportamental do processamento auditivo em indivíduos gagos concluímos, que neste estudo:

A orelha direita apresentou melhor desempenho do que a orelha esquerda nos diferentes testes comportamentais com sons verbais.

O grau de gravidade da gagueira não interferiu com o resultado de cada teste comportamental do processamento auditivo.

\begin{abstract}
Purpose: To compare the difference between the performances of right and left ears in behavioral tests of auditory processing and to compare the results obtained by subjects with different stuttering severity classifications in each auditory processing test. Methods: Fifty six subjects ( 49 male, 7 female), with ages ranging from four to 34 years, were referred to auditory processing evaluation as a complement to speech and language evaluation. All subjects were submitted to auditory, speech and language evaluation. Disfluency severity was classified according to the Riley Stuttering Severity Index as: very mild, mild, moderate, severe and very severe. Behavioral auditory processing tests were selected and analyzed regarding the patients' age and following the proposal of Pereira \& Schochat (1997). Results: Mild stuttering was prevalent among subjects with ages ranging from four to seven years and from 12 to 34 years. Moderate stuttering was the most prevalent degree among subjects with eight to 11 years old. Auditory processing disorders were observed in $92,85 \%$ of the subjects tested. Significant statistical differences between left and right ears were found in directed attention conditions for the nonverbal dichotic test, in all age ranges. No differences were found among subjects with different stuttering severity classifications in any of the auditory processing tests carried out. Conclusions: Right ear showed better results than left ear in both monotic and dichotic tests. Stuttering severity didn't interfere with the results of each test.
\end{abstract}

KEYWORDS: Stuttering; Auditory perceptual disorders; Hearing; Speech disorders

\section{REFERÊNCIAS}

1. Andrade CRF. Processamento da fala - aspectos da fluência. Pró-Fono. 2000;12(1):69-71.

2. Andrade CRF, Juste F. Aplicação de um teste americano de severidade da gagueira (SSI) em crianças fluentes falantes do Português brasileiro. Pró-Fono. 2001;13(2):177-80.

3. Perkins WH, Kent RD, Curlee RF. A theory of neuropsycholinguistic function in stuttering. J Speech Hear Res. 1991;34(4):734-52. Review.

4. Gomes MJC, Scrochio EF. Terapia da gagueira em grupo: experiência a partir de um grupo de apoio ao gago. Rev Bras Ter Comport Cogn. 2001;3(2):25-34.

5. Oliveira AMCC, Ribeiro IM, Merlo S, Chiappetta ALML. O que fonoaudiólogos e estudantes de fonoaudiologia entendem por fluência e disfluência. Rev CEFAC. 2007;9(1):40-6.

6. Blood IM. Disruptions in auditory and temporal processing in adults who stutter. Percept Mot Skills. 1996;82(1):272-4.

7. Kramer MB, Green D, Guitar B. A comparison of stutterers and nonstutterers on masking level differences and synthetic sentence identification tasks. J Commun Disord. 1987;20(5):379-90.

8. Meyers SC, Hughes LF, Schoeny ZG. Temporal-phonemic processing skills in adult stutterers and nonstutterers. J Speech Hear Res. 1989;32(2):274-80.

9. Alvarez AMMA, Balen SA, Misorelli MIL, Sanchez ML. Processamento auditivo central: proposta de avaliação e diagnóstico diferencial. In: Munhoz MSL, Caovilla HH, Silva MLG, Ganança MM, editores. Audiologia clínica. São Paulo: Atheneu; 2000. p. 103-20.

10. Wynne MK, Boehmler RM. Central auditory function in fluent and disfluent normal speakers. J Speech Hear Res. 1982;25(1):54-7.

11. Blood GW, Blood IM. Central auditory function in young stutterers. Percept Mot Skills. 1984;59(3):699-705.

12. Riley GD. A stuttering severity instrument for children and adults. SSI-3. 3rd ed. Austin: ProEd; 1994.

13. Nuck ME, Blood GW, Blood IM. Fluent and disfluent normal speakers' responses on a Synthetic Sentence Identification (SSI) task. J Commun Disord. 1987;20(2):161-9. 
14. Rastatter MP, Dell CW. Reaction times of moderate and severe stutterers to monaural verbal stimuli: some implications for neurolinguistic organization. J Speech Hear Res. 1987;30(1):21-7.

15. Musiek FE, Gollegly KM, Lamb LE, Lamb P. Selected issues in screening for central auditory processing dysfunction. Semin Hear. 1990;11:372-83.

16. Pereira LD, Schochat E. Processamento auditivo central: manual de avaliação. São Paulo: Lovise; 1997.

17. Hall JW, Jerger J. Central auditory function in stutterers. J Speech Hear Res. 1978;21(2):324-37.

18. Schiefer AM, Barbosa LMG, Pereira LD. Considerações preliminares entre uma possível correlação entre a gagueira e os aspectos lingüísticos e auditivos. Pró-Fono. 1999;11(1):27-31.

19. Andrade AN, Gil D, Schiefer AM, Pereira LD. Avaliação do processamento auditivo em indivíduos com queixa de disfluência. In: $20^{\circ}$ Encontro Internacional de Audiologia; 2005, Abril 21-24. Anais. São Paulo; 2005.

20. Bradshaw JL, Nettleton NC. Human cerebral asymmetry. New Jersey: Prentice Hal; 1983.

21. Manning WH, Riensche L. Auditory assembly abilities of stuttering and nonstuttering children. J Speech Hear Res. 1976;19(4):777-83.

22. Pereira LD. Avaliação do processamento auditivo central. In: Lopes Filho OC, editor. Tratado de fonoaudiologia. São Paulo: Roca; 1997. p.109-26.
23. Almeida CC. Semi-alfabetizados versus alfabetizados: avaliação auditiva através dos testes SSW, discriminação e consciência fonológica. [Monografia de Especialização]. São Paulo: Universidade Federal de São Paulo. Escola Paulista de Medicina; 1997.

24. Foundas AL, Corey DM, Hurley MM, Hellman KM. Verbal dichotic listening in developmental stuttering: subgroups with atypical auditory processing. Cogn Behav Neurol. 2004;17(4):224-32.

25. Strub RL, Black FW, Naeser MA. Anamalous dominance in sibling stutterers: evidence from CT scan asymmetries, dichotic listening, neuropsychological testing, and handedness. Brain Lang. 1987;30(2):338-50.

26. Bocca C, Calearo C, Cassinari V, Migliavaca F. Testing "cortical" hearing in temporal lobe tumors. Acta Otolaryngol. 1955;45:289-304.

27. Kimura D. Some effects of temporal-lobe damage on auditory perception. Can J Psychol. 1961; 15:156-65.

28. Berlin CI, Lowe-Bell SS, Cullen JK Jr, Thompson CI. Dichotic speech perception: an interpretation of right-ear advantage and temporal offset effects. J Acoust Soc Am. 1973;53(3):699-709.

29. Curry FK, Gregory HH. The performance of stutterers on dichotic listening tasks thought to reflect cerebral dominance. J Speech Hear Res. 1969;12(1):73-82.

30. Toscher MM, Rupp RR. A study of the central auditory processes in stutterers using the Synthetic Sentence Identification (SSI) Test battery. J Speech Hear Res. 1978;21(4):779-92. 